\title{
Toward an Improved Wind Quality Control for RapidScat
}

\author{
Wenming Lin and Marcos Portabella
}

\begin{abstract}
Quality control (QC) is an essential part of the scatterometer wind retrieval. In the current pencil-beam scatterometer wind processor (PenWP), a maximum likelihood estimator (MLE)-based QC is used to discern between good- and poor-quality winds. MLE QC is generally effective in flagging rain contamination and increased subcell wind variability in the ocean surface wind vectors derived from $\mathrm{Ku}$-band pencilbeam scatterometers, such as the RapidScat (RSCAT) installed on the International Space Station. However, the MLE is not an effective quality indicator over the outer swath where the inversion is underdetermined due to the lack of azimuthal diversity (including lack of horizontal polarized measurements). Besides, it is challenging to discriminate rain contamination from "true" high winds. This paper reviews several wind qualitysensitive indicators derived from the RSCAT data, such as MLE and its spatially averaged value $\left(\mathrm{MLE}_{m}\right)$, and the singularity exponents (SE) derived from an image processing technique, called singularity analysis. Their sensitivities to data quality and rain are evaluated using collocated Advanced Scatterometer wind data, and global precipitation measurement satellite's microwave imager rain data, respectively. It shows that $\mathrm{MLE}_{m}$ and SE are the most effective indicators for filtering the poorest-quality winds over RSCAT inner and outer swath, respectively. A simple combination of $S E$ and $M_{m}$ thresholds is proposed to optimize RSCAT wind QC. Comparing to the operational PenWP QC, the proposed method mitigates over-rejection at high winds, and improves the classification of good- and poor-quality winds.
\end{abstract}

Index Terms-Quality control (QC), rain, scatterometer, singularity analysis (SA), winds.

\section{INTRODUCTION}

$\mathbf{S}$ PACEBORNE scatterometers provide accurate measurements of the normalized radar cross section $\left(\sigma^{0}\right)$ over the sea surface. A set of $\sigma^{0}$ measurements in each wind vector cell (WVC) is inverted into a set of ambiguous wind solutions using a geophysical model function (GMF) that relates wind vector and radar backscatter measurements for a certain observing geometry, polarization, and radar operating frequency [1], [2]. Then a spatial filter, known as ambiguity removal, is used to select one of the ambiguous solutions as the observed wind at every WVC [3], [4]. Generally, the scatterometer-derived winds are of high quality, and widely

Manuscript received September 23, 2016; revised January 24, 2017; accepted March 14, 2017. Date of publication April 12, 2017; date of current version June 22, 2017. This work was supported in part by the European Organization for the Exploitation of Meteorological Satellites Numerical Weather Prediction Satellite Application Facility Associated Scientist under Project NWP-AS16-2 and in part by the Spanish Ministry of Economy and Competitiveness Research and Development under Project ESP2015-67549-C3-2-R.

The authors are with the Institut de Ciències del Mar, 08003 Barcelona, Spain (e-mail: wenminglin@icm.csic.es).

Color versions of one or more of the figures in this paper are available online at http://ieeexplore.iee.org.

Digital Object Identifier 10.1109/TGRS.2017.2683720 used in applications such as nowcasting, short-range forecasting, and numerical weather prediction (NWP) data assimilation among others. However, several geophysical phenomena other than wind, such as rain, wind variability, confused sea state, and land/ice contamination within the radar footprint, can also contribute to the radar backscatter, hence distort the area mean wind-induced $\sigma^{0}$ measurements and degrade the retrieved wind quality [5]-[7]. Note that the presence of rain is a particularly acute problem for $\mathrm{Ku}$-band scatterometer systems [2], [8], such as RapidScat (RSCAT). Consequently, a quality control $(\mathrm{QC})$ is required to discern between goodand poor-quality winds, such that the latter can be filtered out. This is a prerequisite for a successful use of scatterometer data in the above-mentioned applications.

The proposed QC method is developed by analyzing the characteristics of certain quality-sensitive indicators derived from scatterometer data itself, and by optimizing the thresholds of these indicators in order to filter (preserve) as many poor (good) quality winds as possible. A commonly used QC indicator is the inversion residual or maximum likelihood estimator (MLE), which depicts the minimum distance between the backscatter measurements and the scatterometer GMF. A large MLE value corresponds to a large inconsistency between the $\sigma^{0}$ measurements and the GMF, indicating that geophysical conditions other than WVC-mean wind dominate the backscatter signal [2], [8]. For the C-band Advanced Scatterometer (ASCAT), an image processing technique, known as singularity analysis (SA), has proven to be more effective than MLE in terms of detecting rainy WVCs [7], [9]. The singularity exponents (SEs) derived from SA also provide quantitative information about the sub- and/or inter-WVC variability, and is a complementary QC indicator to MLE. The combination of SE and MLE has been proposed to improve the ASCAT wind QC in [10], and to classify and quantify the ASCAT wind uncertainty as a function of the wind variability, based on a triple collocation (TC) analysis of ASCAT, buoy, and the European Centre for MediumRange Weather Forecasting (ECMWF) model output [11]. For Ku-band scatterometers, rain is the most important source of wind quality degradation, and rain detection is thus very relevant [12]-[14]. Normally, rain-contaminated WVCs are simply filtered by setting certain thresholds to MLE and other rainsensitive indicators [15], [16]. However, the MLE is not always sufficiently sensitive to rain contamination effects, e.g., in the outer swath of pencil-beam scatterometers [6], [7], where only the vertical polarized measurements are available and the backscatter set lacks azimuth diversity. Besides, it is difficult to effectively discriminate rain from high winds using MLE, 
since the Ku-band radar backscatter signal is mostly enhanced under rainy conditions, leading to a remarkable increase in the retrieved wind speed. In both cases (outer swath and high winds), it is challenging to reach a compromise between rejecting rain-contaminated data and accepting as many goodquality winds as possible.

This paper aims to find other QC indicators that are more sensitive to the wind quality than MLE, and to improve the RSCAT QC over the outer swath and at high wind conditions. Following the recent progress on ASCAT wind QC, the SE, which is proven to be a good indicator for increased inter-WVC wind variability and thus a good QC indicator, is used to evaluate the quality of Ku-band RSCAT data for the first time. Meanwhile, a new QC indicator, which is simply calculated by averaging the MLE values within a centered $3 \times 3$ box (namely $\mathrm{MLE}_{m}$ ) and thus an additional indicator for inter-WVC variability, is also tested for RSCAT wind QC purposes. Section II describes the derivations of MLE, $\mathrm{MLE}_{m}$, and SE, respectively. In Section III, the sensitivity of these indicators to wind quality is assessed using the collocated ASCAT wind data and the global precipitation measurement satellite's microwave imager (GMI) rain data. In Section IV, a new RSCAT QC is proposed by using SE at the other swath and $\mathrm{MLE}_{m}$ at the inner swath. The effectiveness of this new QC method is verified using collocated moored buoy data. Finally, the conclusions are presented in Section V.

\section{QUALITY INDICATORS}

\section{A. MLE}

The maximum likelihood estimation is the most commonly used method in scatterometer wind retrieval [1], [2]. In the current NWP satellite application facility (SAF) pencil-beam scatterometer wind processor (PenWP), the wind inversion is carried out by minimizing the cost function in the following equation:

$$
\text { MLE }=\frac{1}{N} \sum_{i}^{N} \frac{\left(\sigma_{m i}{ }^{0}-\sigma_{s i}^{0}\right)^{2}}{\left(K_{p i} \cdot \sigma_{m i}^{0}\right)^{2}}
$$

where $\sigma_{m i}^{0}$ is the backscatter measured by the $i$ th beam, $\sigma_{s i}^{0}$ is the corresponding backscatter simulated through the GMF, and $K_{p i}$ is the corresponding measurement error standard deviation (SD). Before the inversion, the backscatter measurements of the egg-shaped footprints are aggregated into four beams, i.e., inner (HH-polarized) fore- and aft-beam, and outer (VV-polarized) fore- and aft-beam. The RSCAT swath is therefore subdivided into inner (WVC numbers 5-37) and outer (WVC numbers 1-4 and 38-41) swaths according to the availability of HH-polarized measurements. In the inner swath, both $\mathrm{HH}$ and VV beams are available, while in the outer swath, only VV-polarized measurements are available. Note that in the outer swath, the VV fore and aft eggs are redistributed over four azimuthal beams as well. Therefore, the number of $\sigma^{0}$ measurements $N$ always equals to four.

The inversion residual of (1), namely, MLE, represents the distance between a measurement $\sigma_{m i}^{0}$ set and its solution $\sigma_{s i}^{0}$ set, and provides information on the consistency among the different backscatter measurements or beams.
It is thus a commonly used indicator for scatterometer wind QC purposes. In practice, the MLE is further normalized by its expected value for a given wind condition and acrosstrack position or WVC number, in order to achieve a uniform behavior and therefore uniform QC metrics [8]. However, the normalized MLE still presents a small dependence on the WVC number and a strong dependence on the wind speed. For example, for SeaWinds onboard QuikSCAT, the following speed-dependent MLE threshold is used to filter poor-quality winds [16]:

$$
\begin{cases}T_{\mathrm{MLE}}=4-0.02 \times(w-5)^{2} & w \leq 15 \mathrm{~m} / \mathrm{s} \\ T_{\mathrm{MLE}}=2 & w>15 \mathrm{~m} / \mathrm{s}\end{cases}
$$

where $w$ is the retrieved wind speed. Assuming that the geophysical uncertainties dominate the degradation of wind quality, it is easy to set the MLE threshold for the other pencil-beam scatterometers (such as RSCAT, Oceansat-II scatterometer (OSCAT) Onboard Oceansat-II Satellite, and Haiyang-2A scatterometer (HSCAT) Onboard HY-2A Satellite) by matching their ratio of QC-rejected data to that of SeaWinds. In PenWP, the MLE dependence on WVC number is also taken into account, such that the actual QC threshold is developed for each WVC number.

\section{B. $S E$}

Following the successful use of SA in improving the ASCAT wind QC, SE is further characterized and adapted for Ku-band scatterometer QC purposes. In particular, a new SA implementation scheme, which combines the gradient measurements of multiple parameters in the analysis, is used to reveal the underlying geophysical phenomena. In this new implementation, SEs are derived using several scatterometerderived parameters (i.e., wind $u, v$ components and MLE) simultaneously as input to SA [17]. SE is estimated through the function in the following equation:

$h(\mathbf{x})=\frac{\log \left[T_{\psi}\|\nabla s\|(\mathbf{x}, r) /\left\langle T_{\psi}\|\nabla s\|(\cdot, r)\right\rangle\right]}{\log r_{0}}+o\left(\frac{1}{\log r_{0}}\right)$

where $T_{\psi}\|\nabla s\|(\mathbf{x}, r)$ is the wavelet projection of the analyzed signal $s$ at the point location $\mathbf{x}$ and the scale factor $r$, $\left\langle T_{\psi}\|\nabla s\|(\cdot, r)\right\rangle$ is the mean value of the wavelet projection over the whole signal. The scale $r_{0}$ is defined as the smallest accessible scale. In the combined SA, $T_{\psi}\|\nabla s\|(\mathbf{x}, r)$ is given by

$$
\begin{aligned}
& T_{\psi}\|\nabla s\| \\
& =\sqrt{\frac{\left(T_{\psi}\|\nabla u\|\right)^{2}}{\left[\sigma\left(T_{\psi}\|\nabla u\|\right)\right]^{2}}+\frac{\left(T_{\psi}\|\nabla v\|\right)^{2}}{\left[\sigma\left(T_{\psi}\|\nabla v\|\right)\right]^{2}}+\frac{\left(T_{\psi}\|\nabla \mathrm{MLE}\|\right)^{2}}{\left[\sigma\left(T_{\psi}\|\nabla \mathrm{MLE}\|\right)\right]^{2}}} .
\end{aligned}
$$

The SDs $\sigma\left(T_{\psi}\|\nabla s\|\right)$ for $u, v$ and MLE fields are estimated from one month of RSCAT 25-km Level 2 (L2) data, as shown in the following equation:

$$
\left\{\begin{array}{l}
\sigma\left(T_{\psi}\|\nabla u\|\right)=0.7 \\
\sigma\left(T_{\psi}\|\nabla v\|\right)=1.2 \\
\sigma\left(T_{\psi}\|\nabla \mathrm{MLE}\|\right)=5.8
\end{array}\right.
$$

and then fixed in our SA software for the entire process. 


\begin{tabular}{|l|l|l|}
\hline 2 & 3 & 2 \\
\hline 3 & 4 & 3 \\
\hline 2 & 3 & 2 \\
\hline
\end{tabular}

Fig. 1. Schematic illustration of the weighting coefficients used to calculate $\mathrm{MLE}_{m}[$ see (6)].

\section{C. $M L E_{m}$}

In general, MLE is a proxy for sub-WVC wind variability, where large positive MLEs are usually found near (gust) fronts, squall lines, and convective systems. The SA-derived $\mathrm{SE}$ is based on spatial derivatives and therefore mostly represents the inter-WVC variability. By using a spatial filter approach similar to the SE derivation, one can also study the inter-WVC variability with the spatially-averaged MLE (i.e., $\mathrm{MLE}_{m}$ ), which may further improve the wind QC. To keep as much subcell wind variability information in the $\mathrm{MLE}_{m}$ as possible, only the eight nearest neighbors of every WVC are used in the spatial averaging. In particular, $\mathrm{MLE}_{m}$ is calculated from a centered $3 \times 3$ box (except at the swath edges, where a $3 \times 2$ box is used) according to the weighting scheme in Fig. 1

$$
\mathrm{MLE}_{m}=\frac{\sum_{i} w_{i} \mathrm{MLE}_{i}}{\sum_{i} w_{i}} .
$$

The $w_{i}$ settings may be optimized for different applications. For QC purposes, the goal is to detect both the sub- and inter-WVC variability, thus the closer the distance to the centered WVC, the higher the weight.

\section{Characterization OF QUALITY INDICATORS}

\section{A. Data}

The first data set consists of six months (January-June 2015) of RSCAT 25-km L2 data collocated with GMI rain data (obtained from the Remote Sensing Systems web site www.remss.com/missions/gmi). The collocation criteria for GMI rain data are less than $10 \mathrm{~min}$ and $0.125^{\circ}$ spatial distance from the RSCAT measurements. The total amount of collocations is about 3.7 million, in which the percentage of rain free (GMI RR $=0 \mathrm{~mm} / \mathrm{h}$ ), light rain (GMI RR $<1 \mathrm{~mm} / \mathrm{h}$ ), moderate and heavy rain (GMI RR $>1 \mathrm{~mm} / \mathrm{h}$ ) data is about $88.1 \%, 8.7 \%$, and $3.2 \%$, respectively. This data set is used to assess the sensitivity of the MLE, $\mathrm{MLE}_{m}$, and SE indicators in terms of flagging rain.

To develop the QC method for Ku-band scatterometer winds, a reliable and adequate wind source is required as reference, particularly under large small-scale wind variability and/or rainy conditions. NWP model (e.g., ECMWF) wind output can be collocated at each WVC by interpolating the model forecasts both spatially and temporally to the scatterometer acquisition location and time, ensuring sufficient collocations over a short period. However, the lowresolution NWP model does not well resolve the wind field at scatterometer scales, i.e., about $25 \mathrm{~km}$, particularly in the presence of rain or high wind variability [7], [11]. Buoy winds are generally accurate. Under high subcell wind variability conditions, the mean buoy wind vectors derived from a series of 10-min discrete buoy measurements have proven to be the most accurate wind source at $25-\mathrm{km}$ scales [11]. However, buoy data are too scarce to assess the mentioned indicator sensitivity to rain and data quality.

Lin et al. [11] show that ASCAT generally provides higher quality winds than ECMWF and buoy data at $25-\mathrm{km}$ scales. Besides, a large amount of well collocated (in space and time) RSCAT and ASCAT wind data can be achieved due to the inclined ISS orbit. Therefore, the second data set consists of RSCAT $25-\mathrm{km} \mathrm{L2}$ data collocated with ASCAT-A $25-\mathrm{km}$ L2 data over the same period as GMI. Both RSCAT and ASCAT data in binary universal format representation are provided by the European Organization for the Exploitation of Meteorological Satellites Ocean and Sea Ice SAF. The collocation criteria for ASCAT data are constrained to be $15 \mathrm{~min}$ and $12.5 \mathrm{~km}$ from the RSCAT measurements. The total number of ASCAT QC-accepted data in this data set is about 1.3 million, while the rejection rate of ASCAT data is about $0.85 \%$.

Moreover, buoy wind collocations are also used to validate the proposed QC algorithm. The buoy data (January-December 2015) are acquired from ECMWF Meteorological Archival and Retrieval System (MARS). The collocation criteria for buoy data are $30 \mathrm{~min}$ distance in time and $25-\mathrm{km}$ distance in space from the RSCAT acquisitions. The total amount of collocations in this data set is about 46 thousand.

\section{B. Analysis}

As in [8] and [16], the MLE presents a slight dependence on across-track position or WVC number and a strong dependence on the retrieved wind speed. Note though that this also happens for the derived SE and $\mathrm{MLE}_{m}$ indicators. The analysis should take such dependence into account. In this paper, the collocated data are separated into inner- and outerswath WVCs, and into 21 speed bins of $1 \mathrm{~m} / \mathrm{s}$ (the last bin includes all $w>20 \mathrm{~m} / \mathrm{s}$ ) for each swath category. The inner swath data are further separated into the sweet and the nadir swath regions in order to check the impact of azimuth diversity on the sensitivity of the proposed indicators to wind quality. This swath separation according to azimuth diversity is commonly used for pencil-beam rotating systems [6], [16]. The sweet region WVCs (WVC numbers 5-15 and 26-37) are sampled with relatively good azimuth diversity, while the nadir region WVCs (WVC numbers 16-25) are sampled with poor azimuth diversity (i.e., separation between fore and aft beams is larger than $150^{\circ}$ ). Assuming that the wind quality or the rain impact is a monotonic function of each quality indicator, the collocated data are then sorted by MLE and $\mathrm{MLE}_{m}$ in descending order, and by SE in ascending order for each of 

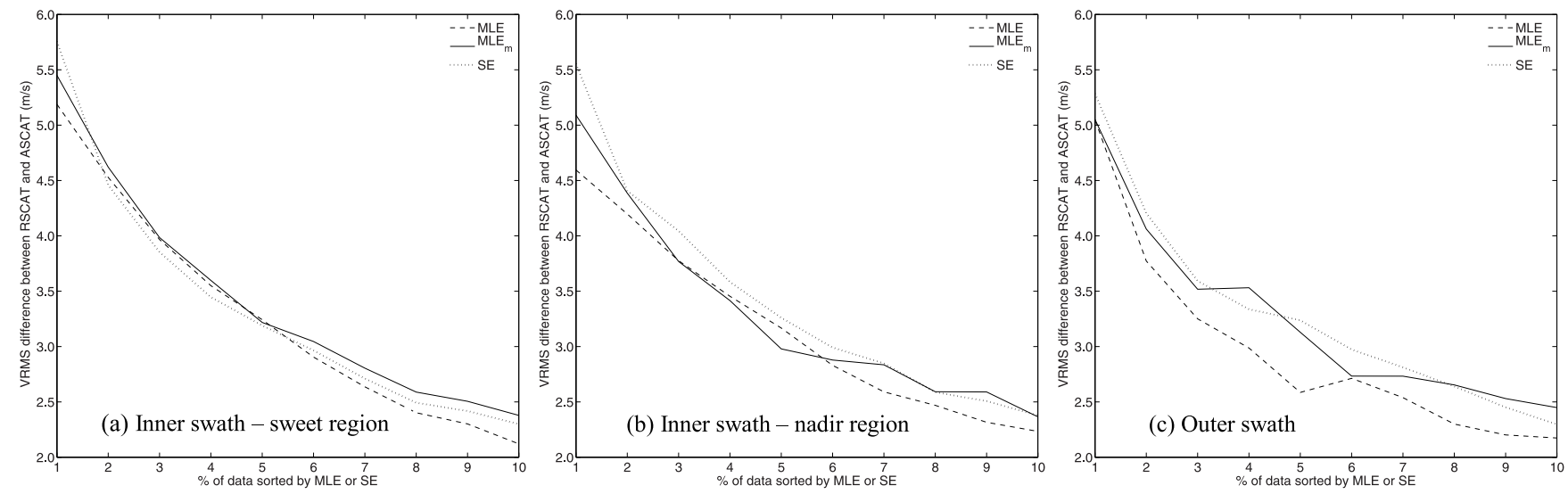

Fig. 2. VRMS difference between RSCAT and ASCAT as a function of the sorted percentiles by MLE, MLE $m$, and SE in the inner swath. (a) Sweet region. (b) Nadir region. (c) Outer swath.

the above mentioned categories/bins. Then each set of sorted data are segregated into a series of $1 \%$ bins in order to compare the sensitivity of these indicators to wind quality or rain in a straightforward way (regardless of their actual values).

The scatterometer wind quality is usually evaluated using the vector root mean square (VRMS) difference between its wind vectors and the reference winds as follows [17]:

$$
\mathrm{VRMS}=\sqrt{\frac{1}{N} \sum_{i}^{N}\left[\left(u_{i}^{\mathrm{scat}}-u_{i}^{\mathrm{ref}}\right)^{2}+\left(v_{i}^{\mathrm{scat}}-v_{i}^{\mathrm{ref}}\right)^{2}\right]}
$$

where $\left(u_{i}^{\text {scat }}, v_{i}^{\text {scat }}\right)$ and $\left(u_{i}^{\text {ref }}, v_{i}^{\text {ref }}\right)$ are the $i$ th scatterometer and reference wind vector, respectively; and $N$ is the number of collocations. ASCAT-derived winds are generally of very high quality and little sensitive to rain contamination effects [11], so they are used as reference in the following analysis. Note though that for increased wind variability and/or very high wind conditions, the quality of all wind sources at $25-\mathrm{km}$ scales is degraded, leading to higher VRMS scores [11]. That is, the RSCAT and ASCAT VRMS scores are actually used to detect when their quality degrades rather than to quantify the inherent quality of RSCAT winds. Fig. 2 shows the VRMS difference between RSCAT and ASCAT winds as a function of the percentiles sorted by MLE (dashed curve), $\mathrm{MLE}_{m}$ (solid curve), and SE (dotted line). Here, the same percentage of data in each speed category is used to construct the general trends of wind quality. In terms of flagging the most discrepant RSCAT and ASCAT winds, the azimuth diversity does impact the sensitivity of the three indicators, notably that of the MLE. In the outer swath, the poor azimuth diversity (similar to that of the nadir swath) is aggravated by the lack of HH-polarized measurements. This in turn degrades the sensitivity of each indicator to wind quality. For the sweet region, the three indicators show their highest sensitivity to wind quality, the $\mathrm{MLE}_{m}$ being slightly more sensitive than SE and MLE. For the nadir region and outer-swath WVCs, $\mathrm{SE}$ is generally the most effective indicator (particularly for the top $3 \%$ of data). $\mathrm{MLE}_{m}$ and SE have similar sensitivity to the data quality, and therefore prove to be remarkably more effective in terms of data quality classification.

Taking the sweet region data as example, Fig. 3 shows the percentage of rain-contaminated data [i.e., with collocated
GMI rain rate (RR) above $1 \mathrm{~mm} / \mathrm{h}$ ] as a function of RSCAT wind speed and the sorted percentiles by MLE, $\mathrm{MLE}_{m}$, and SE, respectively. Actually, such illustrations are similar to those of nadir region (not shown), indicating that the azimuth diversity is not relevant in terms of rain identification for the inner swath WVCs. As expected, the retrieved high winds are more likely to be rain contaminated than the low winds. The operational MLE threshold is converted into the rejection ratio, and plotted as the white dashed curve for reference. This curve well aligns well with a high ratio of rainy data, which indicates that the MLE-based QC is effective in detecting rain. $\mathrm{MLE}_{m}$ though is even more effective than MLE. On the one hand, for the same rejection rate, $\mathrm{MLE}_{m}$ filters more raincontaminated data than MLE [see the larger accumulation of data at left side of the white dashed curve in Fig. 3(b) compared to that of Fig. 3(a)]. On the other hand, $\mathrm{MLE}_{m}$ can be used to identify more rain-free data than MLE [see the larger light-gray area in Fig. 3(b) compared to Fig. 3(a)], particularly at high winds condition. $\mathrm{MLE}_{m}$ and MLE are generally both better than SE for rain detection purpose. However, as shown in Fig. 2, SE is comparable to $\mathrm{MLE}_{m}$ in filtering the most discrepant RSCAT and ASCAT winds. This indicates that other geophysical phenomena besides rain contamination, e.g., increased local wind variability (note that rain induces increased wind variability well beyond the rainy cells [10], [11]), also degrade the RSCAT wind quality. Such phenomena are more evident in the SE field than in the $\mathrm{MLE}_{\mathrm{MLE}} \mathrm{M}_{m}$ fields. Therefore, SE can be a complementary indicator to $\mathrm{MLE} / \mathrm{MLE}_{m}$ in classifying $\mathrm{Ku}$-band wind data quality. Over the outer swath, SE is more sensitive to rain than MLE/MLE $m$, as shown in Fig. 4. Due to the lack of collocations, a thorough analysis of the sensitivity of the $\mathrm{SE} / \mathrm{MLE} / \mathrm{MLE}_{m}$ indicators to rain cannot be carried out in the outer swath. Consequently, Fig. 4 only illustrates the general trends of the quality indicators' sensitivity to rain.

In summary, $\mathrm{MLE}_{m}$ and SE are generally more effective than MLE in flagging the most discrepant RSCAT and ASCAT winds. Moreover, they are the best indicators for rain in the inner- and outer-swath, respectively. For Ku-band scatterometer QC purposes, one may use $\mathrm{MLE}_{m}$ over the inner-swath WVCs and SE over the outer-swath WVCs. This is further discussed in Section IV. 

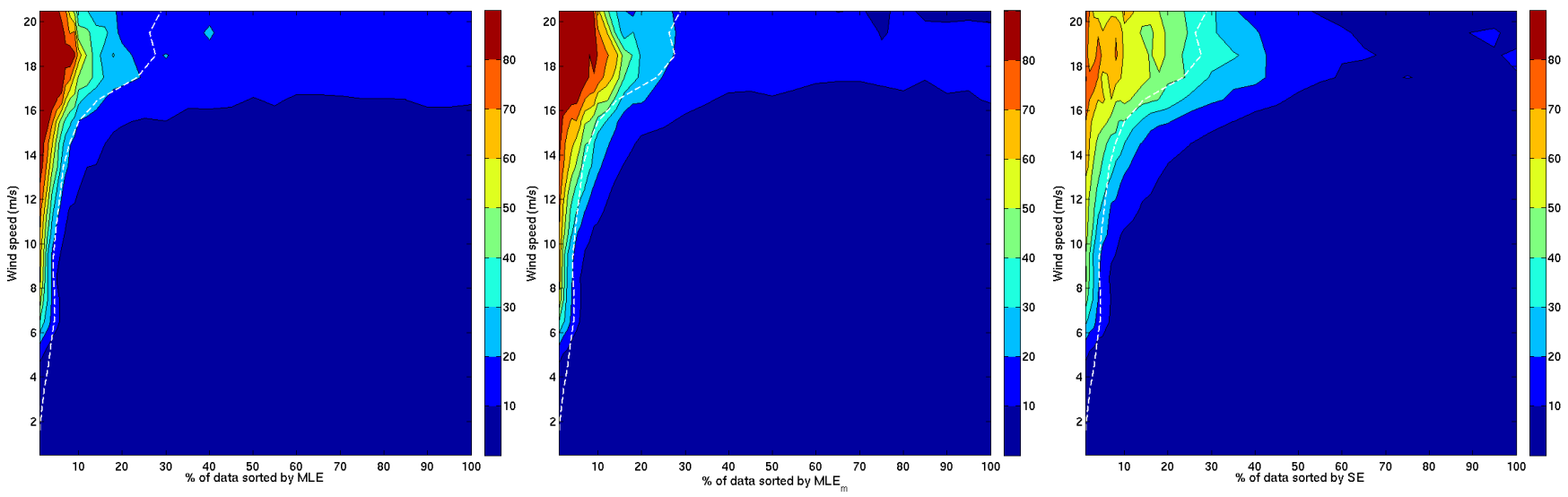

Fig. 3. Percentage of rain-contaminated data (GMI RR $>1 \mathrm{~mm} / \mathrm{h}$ ) as a function RSCAT wind speed and the sorted percentiles by: (a) MLE, (b) MLE $m$, and (c) SE. Only sweet-region WVCs are analyzed. The white dashed curve indicates the rejection ratio of the operational MLE-based QC.

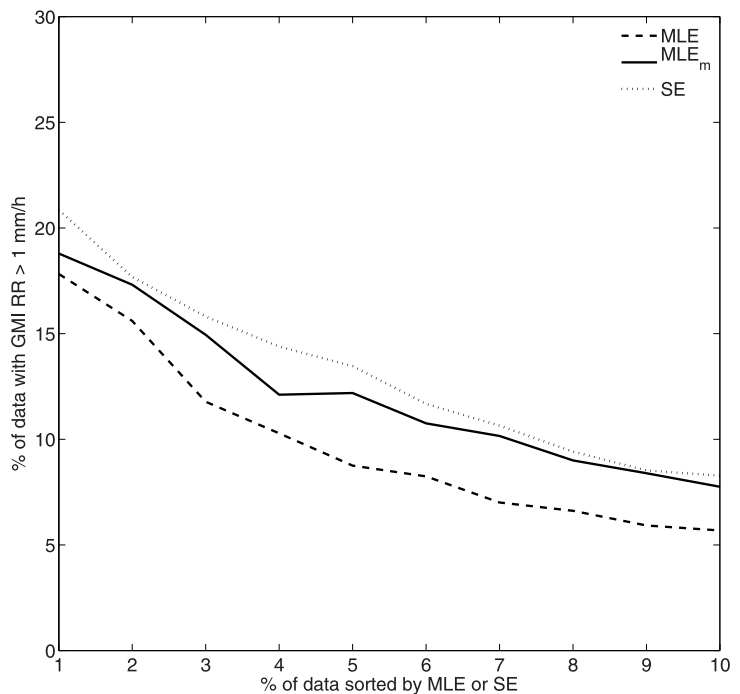

Fig. 4. Percentage of rain-contaminated data (GMI RR $>1 \mathrm{~mm} / \mathrm{h})$ as a function of the sorted percentiles by MLE (dashed curve), $\mathrm{MLE}_{m}$ (solid curve), and SE (dotted curve) for the outer swath WVCs.

\section{VERIFICATION}

The objective of scatterometer QC is to maximize poorquality rejections (including rain) while minimizing goodquality rejections. In other words, given a certain filtering objective (i.e., rejection ratio), the scatterometer QC aims to maximize the error scores (e.g., the VRMS difference between RSCAT and ASCAT winds, using the latter as reference) of the rejected category, and to minimize the error scores of the preserved or accepted category. In this paper, the rejection ratio of QuikSCAT data, which has been well verified in [6] and [16], is used as reference for the RSCAT QC. The QC thresholds for $\mathrm{MLE}_{m}$ and SE are adjusted separately in each collocated data set, such that the overall percentage of QC-rejected data by $\mathrm{MLE}_{m}$ and SE is equivalent to that of the PenWP MLE-based QC in each WVC and wind speed bin. The objective then becomes to get higher VRMS scores than those of the operational QC with a new methodology. Note that, this is not the optimal way to determine the QC thresholds, but a straightforward method to assess the performance of different
QC indicators. Finally, the three QC methods are verified using the collocated GMI, ASCAT, and buoy data, respectively. Since the azimuth separation is not relevant in terms of rain identification for the inner swath WVCs, we do not separate the inner swath into sweet and nadir regions anymore in this section.

\section{A. RSCAT-GMI Analysis}

Table I compares the general performance of the three QC indicators in terms of rain detection. In the inner swath, $\mathrm{MLE}_{m}$ is the best rain indicator, followed by MLE and SE. While in the outer swath, SE performs better than $\mathrm{MLE}_{m}$ in detecting rain. For each column, there is a higher rain detection ratio in the inner swath than in the outer swath. Since the ratio of rainy data (GMI RR $>1 \mathrm{~mm} / \mathrm{h}$ ) is about $3.2 \%$ for both inner and outer swath, it is inferred that the rain detectability is higher in the inner swath than in the outer swath.

Fig. 5 assesses the rain detection ratio as a function of wind speed particularly. In the inner swath [Fig. 5(a)], the $\mathrm{MLE}_{m}$ indicator has the highest rain detection ratio among all three, for moderate and high winds $(w>7 \mathrm{~m} / \mathrm{s})$. This implies that the $\mathrm{MLE}_{m}$ can be used to preserve more moderate and high winds than the other two indicators while preserving the same rain detection skill, which in turn improves the moderate and high winds QC remarkably. It also shows that SE detects the lowest amount of rain data data for $w<16 \mathrm{~m} / \mathrm{s}$ among the three indicators. Over the outer swath, both $\mathrm{MLE}_{m}$ and notably SE QC show higher rain detection ratio than MLE. Moreover, Fig. 5(b) demonstrates that SE can be the most effective RSCAT QC indicator over the outer swath.

\section{B. RSCAT-ASCAT Analysis}

Table II summarizes the VRMS difference between RSCAT and ASCAT winds for the accepted/rejected categories defined by MLE and the two new QC indicators, i.e., $\mathrm{MLE}_{m}$ and SE. Generally, $\mathrm{MLE}_{m}$ and SE QC are more effective than MLE in filtering the most discrepant RSCAT winds w.r.t. ASCAT winds. As discussed in Section III-B, the high VRMS values for the SE-rejected wind category (see Table II) are probably due to the increased wind variability at moderate and low 
TABLE I

Percentage of Rain Contaminated Data (With GMI RR $>0$ or $1 \mathrm{~mm} / \mathrm{h}$ ) Over THe QC-ReJected Data By Different QC INDiCATors

\begin{tabular}{|c|c|c|c||c|c|c|}
\hline \multirow{3}{*}{ Swath } & \multicolumn{3}{|c||}{$\begin{array}{c}\text { Percentage of GMI RR }>0 \mathrm{~mm} / \mathrm{h} \text { over the QC } \\
\text { rejected data [\%] }\end{array}$} & \multicolumn{3}{c|}{$\begin{array}{c}\text { Percentage of GMI RR }>1 \mathrm{~mm} / \mathrm{h} \text { over the QC } \\
\text { rejected data [\%] }\end{array}$} \\
\cline { 2 - 7 } & MLE & MLE $_{\mathrm{m}}$ & $\mathrm{SE}$ & MLE & MLE $_{\mathrm{m}}$ & $\mathrm{SE}$ \\
\hline Inner & 64.4 & 68.0 & 61.0 & 38.5 & 41.5 & 35.0 \\
\hline Outer & 29.3 & 31.0 & 36.3 & 13.4 & 14.7 & 17.0 \\
\hline
\end{tabular}
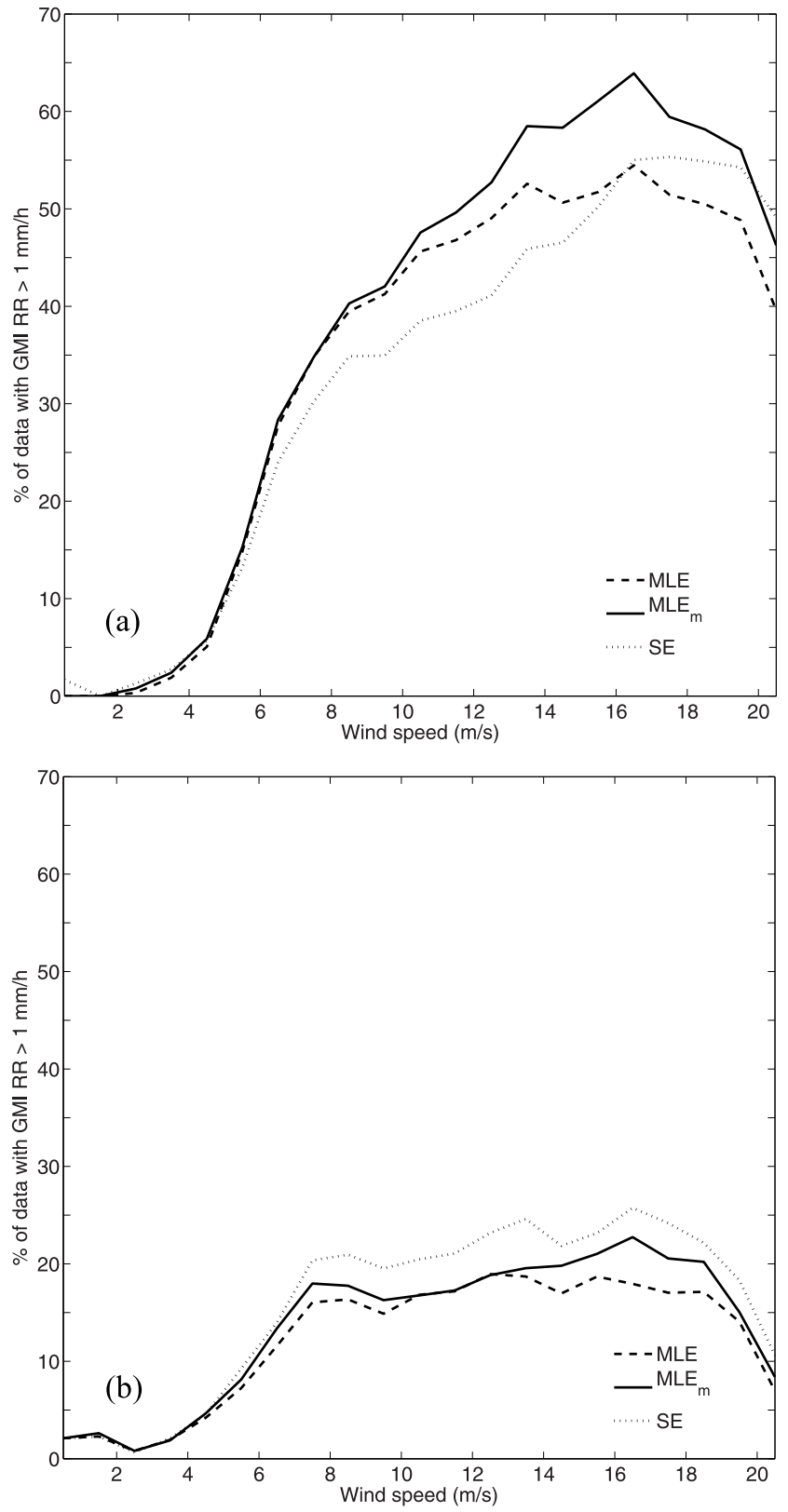

Fig. 5. Percentage of rainy data with GMI RR $>1 \mathrm{~mm} / \mathrm{h}$ over the QC-rejected data set by MLE (dashed curve), MLE $_{m}$ (solid curve), and SE (dotted curve) for the (a) inner swath and (b) outer swath.

winds. These results are in accordance with the conclusions from Fig. 2. They suggest that instead of the operational MLE-based QC, one may use the $\mathrm{MLE}_{m}$ flag over the inner swath and the SE flag over the outer swath to accept the closest RSCAT winds to the (collocated) ASCAT winds.
Fig. 6 shows contour plots of RSCAT versus ASCAT winds over the (RSCAT) inner swath for different flag combinations. The data rejected by the $\mathrm{MLE}_{m}$ QC but accepted by the MLE QC are probably contaminated by rain, as indicated by the remarkable RSCAT wind speed bias w.r.t. ASCAT in Fig. 6(a). When the data are preserved by the $\mathrm{MLE}_{m}$ QC but rejected by the MLE QC, there is a better correspondence between RSCAT and ASCAT winds, as shown in Fig. 6(b). Note that the RSCAT wind speed bias is smaller than in Fig. 6(a) and only present at low and medium winds (below $10 \mathrm{~m} / \mathrm{s}$ ). Consequently, the current PenWP QC can be simply improved by using $\mathrm{MLE}_{m}$ instead of MLE in this case. SE is less sensitive to rain than MLE or $\mathrm{MLE}_{m}$. The relatively high VRMS and low bias of the SE QC-rejected and MLE QC-accepted [Fig. 6(c)] data, and in particular of SE QC-rejected and $\mathrm{MLE}_{m}$ QC-accepted (not shown) data, indicates that such data are less likely to be rain contaminated but more likely to be associated with high subcell wind variability, e.g., over strong convergence or divergence areas. Fig. 6(c) demonstrates that the wind speed retrieval is of fair quality for such variable winds (see also the statistic of wind direction below). Despite the slightly positive bias at high winds, which is probably due to some residual rain contamination, it may be essential to keep the variable winds since they provide useful information on the air-sea interaction processes for some applications, such as nowcasting and oceanography. However, for other applications such as data assimilation, variable winds are a potential risk [18], [19]. Thus the SE QC should be applied with caution. For reference, the RSCAT wind speed versus ASCAT wind speed for the data accepted by both MLE and $\mathrm{MLE}_{m}$ QC are shown in Fig. 6(d). As expected, RSCAT and ASCAT winds are in very good agreement for such category, which corresponds to mostly rain free and low wind variability conditions. Moreover, the RSCAT versus ASCAT wind directions (not shown) have the following SD values: $26.8^{\circ}, 22.7^{\circ}, 28.5^{\circ}$, and $18.4^{\circ}$ for the same flag combinations as in Fig. 6(a)-(d), respectively. Similar conclusions to those from Fig. 6 can be drawn from the wind direction statistics.

Over the outer swath, a similar study as above shows that the SE-rejected RSCAT winds have the highest speed bias w.r.t. ASCAT winds (not shown). And the SDs of the difference between RSCAT and ASCAT wind directions are $21.8^{\circ}, 19.9^{\circ}, 26.7^{\circ}$, and $18.8^{\circ}$, respectively, for the four QC flag combinations. Although the wind quality of the outer-swath WVCs is generally lower than that of the innerswath WVCs, SE is shown to be the most effective indicator among the three in terms of wind QC. 
TABLE II

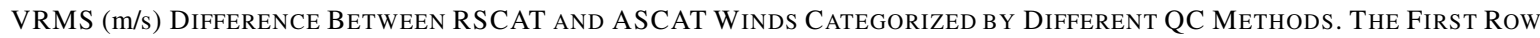
SHOWs THE Statistics For INNER-SWATH WVCs, AND THE SECOND ROW FOR THE OUTER-SWATH WVCs

\begin{tabular}{|c|c|c||c|c||c|c|}
\hline \multirow{3}{*}{ Swath } & \multicolumn{2}{|c||}{ MLE } & \multicolumn{2}{c||}{ MLE $_{\mathrm{m}}$} & \multicolumn{2}{c|}{ SE } \\
\cline { 2 - 7 } & \multicolumn{2}{|c||}{ VRMS [m/s] } & \multicolumn{2}{c|}{ VRMS [m/s] } & \multicolumn{2}{c|}{ VRMS [m/s] } \\
\cline { 2 - 7 } & Accept & Reject & Accept & Reject & Accet & Reject \\
\hline Inner & 1.55 & 4.14 & 1.53 & 4.25 & 1.53 & 4.26 \\
\hline Outer & 1.67 & 3.39 & 1.65 & 3.56 & 1.63 & 3.75 \\
\hline
\end{tabular}
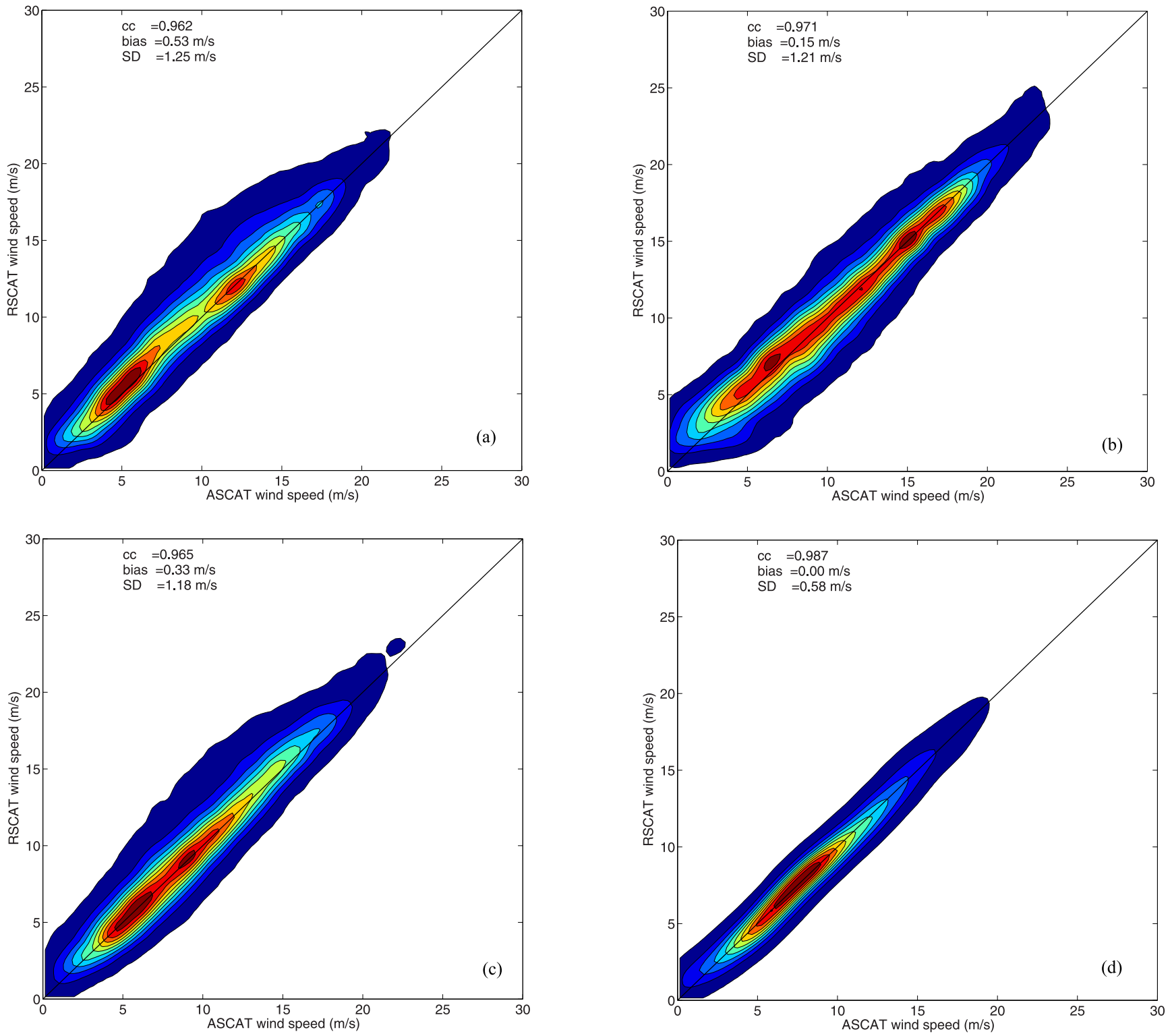

Fig. 6. RSCAT versus ASCAT wind speeds for the (RSCAT) inner swath and the following flag combinations: (a) fMLE $=0$, and fMLE $m=1$; (b) $\mathrm{fMLE}=1$, and $\mathrm{fMLE}_{m}=0$; (c) fMLE $=0$, and $\mathrm{fSE}=1$; and (d) fMLE $=0$, and $\mathrm{fMLE}_{m}=0$. Here the flag values " 0 " and " 1 " indicate QC-accepted and QC-rejected data, respectively. The upper left corner of each panel shows the correlation coefficient (cc), the wind speed bias, and the SD of RSCAT w.r.t. ASCAT winds.

\section{RSCAT-Buoy Analysis}

Following the results in Sections IV-A and IV-B, the implementation of the $\mathrm{MLE}_{m}$ QC over the RSCAT inner swath and the SE QC over the outer swath is proposed. Table III shows the statistics of RSCAT winds versus buoy winds for the different combinations of the MLE QC and the new QC (denoted as "fNEW") flags. The proposed method rejects a similar amount of data as the MLE QC (about 6.6\%, but with significantly more discrepant winds w.r.t. buoy winds (see the larger bias, 
TABLE III

STATISTICS OF RSCAT Winds VERSus BuOY Winds FOR THE DiFFERENT COMBINATIONS OF THE PenWP MLE-BASED QC AND THE

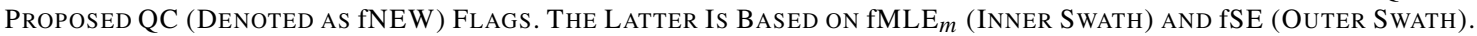
The Statistics Below CorRespond to: Wind SPEed BiAs (m/s), $a b b r . B_{\mathrm{S}}$; SD OF Wind SPEED (m/s), $a b b r . S D_{\mathrm{s}}$; SD of Wind Direction [DEGREe, ${ }^{\circ}$ ], $a b b r . S D_{\mathrm{d}}$; AND THE PERCENTAGE of DATA [\%], $a b b r . P$

\begin{tabular}{|c|c|c|c|c|c|c|c|c|c|c|}
\hline & \multicolumn{4}{|c|}{$\mathrm{fNEW}=0$} & \multicolumn{5}{c|}{$\mathrm{fNEW}=1$} \\
\cline { 2 - 12 } & $\begin{array}{c}B_{\mathrm{s}} \\
(\mathrm{m} / \mathrm{s})\end{array}$ & $\begin{array}{c}S D_{\mathrm{s}} \\
(\mathrm{m} / \mathrm{s})\end{array}$ & $S D_{\mathrm{d}}\left({ }^{\circ}\right)$ & $\begin{array}{c}\text { VRMS } \\
(\mathrm{m} / \mathrm{s})\end{array}$ & $P(\%)$ & $\begin{array}{c}B_{\mathrm{s}} \\
(\mathrm{m} / \mathrm{s})\end{array}$ & $\begin{array}{c}S D_{\mathrm{s}} \\
(\mathrm{m} / \mathrm{s})\end{array}$ & $S D_{\mathrm{d}}\left({ }^{\circ}\right)$ & $\begin{array}{c}\text { VRMS } \\
(\mathrm{m} / \mathrm{s})\end{array}$ & $P(\%)$ \\
\hline $\mathrm{fMLE}=0$ & -0.04 & 1.07 & 18.1 & 2.25 & 91.0 & 0.73 & 1.86 & 33.6 & 4.43 & 2.4 \\
\hline $\mathrm{fMLE}=1$ & 0.40 & 1.69 & 27.8 & 3.60 & 2.4 & 2.30 & 3.06 & 40.6 & 6.67 & 4.2 \\
\hline
\end{tabular}

TABLE IV

CALIBRATION COEFFICIENTS AND THE INHERENT SD ERRORS OF RSCAT Wind COMPONENTS ESTIMATED FROM A TC ANALYSiS. THE SD ERrors ARE Estimated ON THE SCALE RESOLVED By THE SCATTEROMETER

\begin{tabular}{|c|c|c|c|c|c|c|c|c|}
\hline & \multicolumn{3}{|c|}{$\mathrm{fNEW}=0$} & \multicolumn{3}{c|}{$\mathrm{fNEW}=1$} \\
& \multicolumn{3}{|c|}{$\begin{array}{c}\text { Calibration } \\
\text { coefficients }\end{array}$} & $\begin{array}{c}\text { Estimated SD errors } \\
{[\mathrm{m} / \mathrm{s}]}\end{array}$ & \multicolumn{2}{c|}{$\begin{array}{c}\text { Calibration } \\
\text { coefficients }\end{array}$} & $\begin{array}{c}\text { Estimated SD errors } \\
{[\mathrm{m} / \mathrm{s}]}\end{array}$ \\
\cline { 2 - 9 } & $u$ & $v$ & $u$ & $v$ & $u$ & $v$ & $u$ & $v$ \\
\hline $\mathrm{fMLE}=0$ & 0.98 & 0.99 & 0.73 & 0.77 & 0.90 & 0.88 & 1.3 & 1.5 \\
\hline $\mathrm{fMLE}=1$ & 0.94 & 0.95 & 1.1 & 1.1 & 0.76 & 0.74 & 2.1 & 2.3 \\
\hline
\end{tabular}

$\mathrm{SD}$, and VRMS values for $\mathrm{fMLE}=0$ and $\mathrm{fNEW}=1$, compared to fMLE $=1$ and fNEW $=0$ ) and ECMWF (not shown). Similar to Fig. 6, the flag values "0" and "1" indicate QC-accepted and QC-rejected data, respectively. A TC analysis is performed to further assess the errors of RSCAT winds in the four categories of Table III. Given a set of TCs (in this case buoy, RSCAT and ECMWF winds) and assuming linear calibration, one can calculate the independent errors of the three wind sources and the calibration coefficients simultaneously [20]. As [20] and [21], the key parameter of TC analysis is the spatial representativeness error, namely, $r^{2}$ value. However, the $r^{2}$ does not affect the calibration coefficients of RSCAT data if one chooses the buoy data as calibration reference [11]. More interesting, the $r^{2}$ value does not affect the estimated errors of buoy and RSCAT data on the scale resolved by the scatterometer $(\sim 25 \mathrm{~km})$. Setting $r^{2}=0$ in the TC analysis, one can easily estimate the calibration coefficients of RSCAT winds versus buoy winds, as well as the inherent scatterometer wind errors, as shown in Table IV. It is confirmed that the new QC is more effective than the operational one in flagging poor quality data.

\section{CONCLUSION}

This paper investigates the correlation between the Ku-band RSCAT wind quality and several scatterometer-derived indicators, such as the conventional quality indicator MLE and its spatially averaged value $\mathrm{MLE}_{m}$, and the SE derived from the retrieved wind and MLE fields. $\mathrm{MLE}_{m}$ proves to be generally more effective than MLE and SE in classifying scatterometer wind data quality and identifying rain contamination over the inner-swath WVCs. However, due to the lack of azimuthal diversity (including the lack of HH-polarized measurements), the inversion problem is not overdetermined in the outer-swath
WVCs, and in turn, both the MLE and $\mathrm{MLE}_{m}$ are not effective noise (and therefore QC) indicators [16]. SE, however, proves to be a more effective QC indicator in the outer swath than $\mathrm{MLE} / \mathrm{MLE}_{m}$, and therefore used for QC purposes in this swath region.

The sensitivity of each indicator to wind quality is given by its effectiveness to detect the most discrepant RSCAT and ASCAT winds (and/or detect rain) for a fixed rejection ratio. The PenWP QC rejection ratio is used as reference and its corresponding VRMS scores are used as benchmark. A set of $\mathrm{MLE}_{m}$ and SE QC thresholds are derived following the same strategy as that used to develop the MLE QC threshold. The rejected data by the three QC methods are then evaluated using different reference data. It is confirmed that the high RSCAT versus ASCAT VRMS and bias scores of the $\mathrm{MLE}_{m}$ QC-rejected and MLE QC-accepted category are mostly attributed to rain (both rain contamination and raininduced wind variability), while the low bias and high VRMS scores of the SE QC-rejected and MLE QC-accepted data are mainly due to increased subcell wind variability. In fact, as concluded in [11], the rejection may depend on the application of the RSCAT-derived winds. For example, cases of rain-induced wind variability may actually be very useful for applications, such as nowcasting and oceanography applications. However, for other applications such as data assimilation into global NWP, variable winds are a potential risk and should therefore be filtered out. This is because global NWP models do not resolve wind variability below 100-200 km scales [21], and as such, rain-induced wind variability as depicted by scatterometers can negatively impact NWP when assimilated. A dedicated study is actually recommended in order to define the most suitable rejection ratio for each application. Finally, an improved RSCAT QC is proposed by using the $\mathrm{MLE}_{m}$ 
over the inner swath and the SE over the outer swath. The assessment using collocated buoy data further validates this conclusion.

The methodologies presented in this paper can be applied to other Ku-band pencil-beam scatterometers, such as the HSCAT onboard HY-2A satellite. In particular, the different combination of MLE, $\mathrm{MLE}_{m}$, and SE QC flags may be used to classify the scatterometer data quality. Future work will focus on adapting the RSCAT QC algorithm for HSCAT QC purposes, and to verify whether the combination of $M_{\mathrm{L}} \mathrm{E}_{\mathrm{m}}$ and $\mathrm{SE}$ is in general optimal for quality controlling other Ku-band rotating pencil-beam scatterometers.

\section{ACKNOWLEDGMENT}

The buoy data were retrieved from the ECMWF MARS archive http://www.ecmwf.int/en/forecasts/datasets. The authors would like to thank A. Verhoef for sharing monthly buoy blacklists and the three anonymous reviewers who helped in improving the quality of this paper.

\section{REFERENCES}

[1] W. J. Pierson, "Probabilities and statistics for backscatter estimates obtained by a scatterometer," J. Geophys. Res., Ocean., vol. 94, no. C7, pp. 9743-9759, Jul. 1989

[2] A. Stoffelen and D. Anderson, "Scatterometer data interpretation: Measurement space and inversion," J. Atmos. Ocean. Techn., vol. 14, no. 6, pp. 1298-1313, Dec. 1997.

[3] J. Vogelzang, A. Stoffelen, A. Verhoef, J. De Vries, and H. Bonekamp, "Validation of two-dimensional variational ambiguity removal on SeaWinds scatterometer data," J. Atmos. Ocean. Techn., vol. 26, no. 7, pp. 1229-1245, Jul. 2009

[4] B. W. Stiles, B. D. Pollard, and R. S. Dunbar, "Direction interval retrieval with thresholded nudging: A method for improving the accuracy of QuikSCAT winds," IEEE Trans. Geosci. Remote Sens., vol. 40, no. 1, pp. 79-89, Jan. 2002.

[5] B. W. Stiles and R. S. Dunbar, "A neural network technique for improving the accuracy of scatterometer winds in rainy conditions," IEEE Trans. Geosci. Remote Sens., vol. 48, no. 8, pp. 3114-3122, Aug. 2010.

[6] M. Portabella and A. Stoffelen, "A comparison of KNMI quality control and JPL rain flag for SeaWinds," Can. J. Remote Sens., vol. 28, no. 3, pp. 424-430, 2002.

[7] M. Portabella et al., "Rain effects on ASCAT-retrieved winds: Toward an improved quality control," IEEE Trans. Geosci. Remote. Sens., vol. 50, no. 7, pp. 2495-2506, Jul. 2012.

[8] M. Portabella and A. Stoffelen, "Characterization of residual information for SeaWinds quality control," IEEE Trans. Geosci. Remote. Sens., vol. 40, no. 12, pp. 2747-2759, Dec. 2002.

[9] W. Lin, M. Portabella, A. Stoffelen, A. Turiel, and A. Verhoef, "Rain identification in ASCAT winds using singularity analysis," IEEE Geosci. Remote Sens. Lett., vol. 11, no. 9, pp. 1519-1523, Sep. 2014.

[10] W. Lin, M. Portabella, A. Stoffelen, A. Verhoef, and A. Turiel, "ASCAT wind quality control near rain," IEEE Trans. Geosci. Remote Sens., vol. 53, no. 8, pp. 4165-4177, Aug. 2015.

[11] W. Lin, M. Portabella, A. Stoffelen, J. Vogelzang, and A. Verhoef, "ASCAT wind quality under high subcell wind variability conditions," J. Geophys. Res., Oceans, vol. 120, pp. 5804-5819, Aug. 2015.
[12] C. Nie and D. G. Long, "A C-band wind/rain backscatter model," IEEE Trans. Geosci. Remote Sens., vol. 45, no. 3, pp. 621-631, Mar. 2007.

[13] D. E. Weissman and M. A. Bourassa, "Measurements of the effect of rain-induced sea surface roughness on the QuikSCAT scatterometer radar cross section," IEEE Trans. Geosci. Remote Sens., vol. 46, no. 10, pp. 2882-2894, Oct. 2008

[14] B. W. Stiles and S. H. Yueh, "Impact of rain on spaceborne Ku-band wind scatterometer data," IEEE Trans. Geosci. Remote Sens., vol. 40 no. 9, pp. 1973-1983, Sep. 2002.

[15] J. N. Huddleston and B. W. Stiles, "A multidimensional histogram rain-flagging technique for SeaWinds on QuikSCAT," in Proc. IEEE Int. Geosci. Remote Sens. Symp., Honolulu, Hawaii, vol. 3. Jul. 2000, pp. $1232-1234$

[16] M. Portabella and A. Stoffelen, "Rain detection and quality control of SeaWinds," J. Atmos. Ocean. Techn., vol. 18, no. 7, pp. 1171-1183, 2001.

[17] W. Lin, M. Portabella, A. Turiel, A. Stoffelen, and A. Verhoef, "An improved singularity analysis for ASCAT wind quality control: Application to low winds," IEEE Trans. Geosci. Remote. Sens., vol. 54, no. 7, pp. 3890-3898, Jul. 2016.

[18] G. D. Chiara, P. Janssen, J.-R. Bidlot, and S. English, "Impact of studies of ASCAT winds in the ECMWF 4D-Var assimilation system," in Proc. 12th Int. Winds Workshop, (IWW), Copenhagen, Denmark, Jun. 2014, p. S6.01 1-8. [Online]. Available: http://www.eumetsat.int/ website/home/News/ConferencesandEvents/DAT_2441511.html?lang= EN

[19] A. Stoffelen et al., "Scatterometer mesoscale NWP data assimilation," in Proc. 12th Int. Winds Workshop (IWW) Splinter discussion session summary, Copenhagen, Denmark, Jun. 2014, pp. 15-20. [Online]. Available: http://www.eumetsat.int/website/home/News/ConferencesandEvents/ DAT_2441511.html?lang=EN.

[20] A. Stoffelen, "Toward the true near-surface wind speed: Error modeling and calibration using triple collocation," J. Geophys. Res., vol. 103, pp. 7755-7766, Apr. 1998.

[21] J. Vogelzang, A. Stoffelen, A. Verhoef, and J. Figa-Saldña, "On the quality of high-resolution scatterometer winds," J. Geophys. Res., vol. 116, no. C10, p. C10033, 2011.

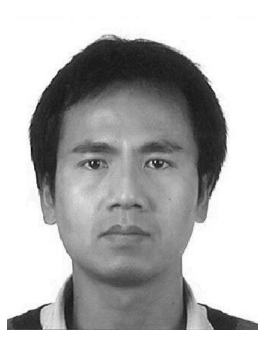

Wenming Lin was born in China in 1984. $\mathrm{He}$ received the B.Sc. degree in engineering from Wuhan University, Wuhan, China, in 2006, and the $\mathrm{Ph} . \mathrm{D}$. degree in engineering from the National Space Science Center, Chinese Academy of Sciences, Beijing, China, in 2011.

$\mathrm{He}$ is currently a Post-Doctoral Researcher with the Institut de Ciències del Mar, Barcelona, Spain, involved in the advanced oceanographic data processing methods, remote sensing of ocean surface winds, and data assimilation.

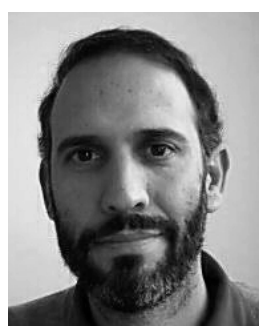

Marcos Portabella was born in Spain in 1970. $\mathrm{He}$ received the B.Sc. degree in physics from the University of Barcelona, Barcelona, Spain, in 1994, the M.Sc. degree in remote sensing from the Institute of Space Studies of Catalonia, Barcelona, in 1995, and the Ph.D. degree in physics from the University of Barcelona.

$\mathrm{He}$ is currently with the Institut de Ciències del Mar, Barcelona, involved in satellite remote sensing, and particularly in scatterometry and L-band radiometry. 